\title{
Insertion and deletion polymorphisms of the ancient AluS family in the human genome
}

Maria S. Kryatova ${ }^{1,2+}$, Jared P. Steranka ${ }^{1,2+}$, Kathleen H. Burns ${ }^{1,2^{*}}$ and Lindsay M. Payer ${ }^{{ }^{*}}$

\begin{abstract}
Background: Polymorphic Alu elements account for $17 \%$ of structural variants in the human genome. The majority of these belong to the youngest AluY subfamilies, and most structural variant discovery efforts have focused on identifying Alu polymorphisms from these currently retrotranspositionally active subfamilies. In this report we analyze polymorphisms from the evolutionarily older AluS subfamily, whose peak activity was tens of millions of years ago. We annotate the AluS polymorphisms, assess their likely mechanism of origin, and evaluate their contribution to structural variation in the human genome.

Results: Of 52 previously reported polymorphic AluS elements ascertained for this study, 48 were confirmed to belong to the AluS subfamily using high stringency subfamily classification criteria. Of these, the majority $(77 \%, 37 / 48)$ appear to be deletion polymorphisms. Two polymorphic AluS elements (4\%) have features of non-classical Alu insertions and one polymorphic AluS element (2\%) likely inserted by a mechanism involving internal priming. Seven AluS polymorphisms (15\%) appear to have arisen by the classical target-primed reverse transcription (TPRT) retrotransposition mechanism. These seven TPRT products are 3' intact with 3' poly-A tails, and are flanked by target site duplications; L1 ORF2p endonuclease cleavage sites were also observed, providing additional evidence that these are L1 ORF2p endonucleasemediated TPRT insertions. Further sequence analysis showed strong conservation of both the RNA polymerase III promoter and SRP9/14 binding sites, important for mediating transcription and interaction with retrotransposition machinery, respectively. This conservation of functional features implies that some of these are fairly recent insertions since they have not diverged significantly from their respective retrotranspositionally competent source elements.

Conclusions: Of the polymorphic AluS elements evaluated in this report, 15\% (7/48) have features consistent with TPRT-mediated insertion, thus suggesting that some AluS elements have been more active recently than previously thought, or that fixation of AluS insertion alleles remains incomplete. These data expand the potential significance of polymorphic AluS elements in contributing to structural variation in the human genome. Future discovery efforts focusing on polymorphic AluS elements are likely to identify more such polymorphisms, and approaches tailored to identify deletion alleles may be warranted.
\end{abstract}

Keywords: Retrotransposon, Mobile element, SINE, Alu, AluS, Polymorphism, Structural variation, Mobilome

\footnotetext{
*Correspondence: kburns@jhmi.edu; Ihorvat1@jhmi.edu

†Equal contributors

'Department of Pathology, Johns Hopkins University School of Medicine, Miller Research Building (MRB) Room 447, 733 North Broadway, Baltimore, MD 21205, USA

Full list of author information is available at the end of the article
} 


\section{Background}

While we have long appreciated differences between individual genomes, it is only recently that robust sequencing efforts have allowed us to begin to build a comprehensive catalog of human structural variants $[1,2]$. Mobile element insertions are an important source of structural variation in the human genome, with Alu elements specifically accounting for $17 \%$ of structural variants $[2,3]$. Alu elements are non-autonomous retrotransposons, relying on the protein machinery of Long INterspersed Element-1 (LINE-1, L1) for their propagation [4]. Classically, new $A l u$ insertions occur by target-primed reverse transcription (TPRT). This mechanism of insertion requires the L1 encoded protein ORF2p, which contains an endonuclease domain and reverse transcriptase domain [4-6]. L1 ORF2p endonuclease has a preference to cleave the negative strand at 5' TTTT/AA 3' sites, but is capable of targeting a range of sequences [7-10]. The T-rich sequence on the cleaved negative strand then primes with the poly-A tail of the Alu transcript, allowing reverse transcriptase to synthesize a copy of the Alu [3]; premature termination of reverse transcription results in the integration of a $5^{\prime}$ truncated element. Because the positive strand is nicked downstream of the initial cleavage site, the newly integrated $A l u$ element is flanked by direct repeats, resulting from a duplication of the sequence at the insertion site when the staggered break is repaired [3]. Thus, an Alu insertion having arisen by TPRT exhibits the following defining features [11]: (1) an intact 3' end, (2) a 3' poly-A tail, and (3) flanking target site duplications (TSDs).

Only a small subset of the 1.1 million $A l u$ insertions in the human genome are capable of retrotransposition, and recent retrotransposition events have created thousands of polymorphic insertions [1, 3, 11-14]. Polymorphic Alu elements almost exclusively belong to the youngest AluY subfamilies $[2,3,7,11,14,15]$. While there have been reports of polymorphic elements from the evolutionarily older AluS subfamily in humans $[2,13,15,16]$, polymorphic AluS insertions are generally not considered to be an important contributor to structural variation and most structural variant discovery efforts have not specifically focused on identifying these elements. In this report we present examples of polymorphic AluS elements, provide annotations of the sequences, and consider the mechanisms that likely created the polymorphisms. Thus, our work expands the potential significance of $A l u S$ elements in contributing to structural variation in the human genome and emphasizes the importance of identifying additional AluS polymorphisms.

\section{Results}

Identification of polymorphic AluS elements in the human genome

How retrotransposon variants in the human genome affect gene expression or phenotype remains poorly elucidated. To better understand the functional effects of these elements, we focus on polymorphic elements near loci associated with disease risk and pathogenesis [17]. We compiled a catalog of previously reported polymorphic Alu elements (see Methods) and from this list selected $112 \mathrm{Alu}$ variants that map near genome-wide association study (GWAS) signals to Sanger sequence and fully annotate [17]. As expected, most (96\%), are from the youngest Alu subfamilies, 46\% AluYa5 and 23\% $A l u Y b 8$, the most recently retrotranspositionally active subfamilies whose members account for the overwhelming majority of previously reported polymorphic Alu insertions [3]. Intriguingly though, $4 \%(n=4)$ belong to the evolutionarily older AluS subfamily, which was most active $35-60$ million years ago $[18,19]$ and is considered to have limited in vivo retrotransposition capability in humans in the modern era [7]. These results suggest that polymorphic AluS elements may contribute to structural variation in the human genome more than previously thought.

Structural variants involving Alu elements may either be deletion or insertion polymorphisms. Since the AluS subfamily is considered to have been largely inactive for tens of millions of years $[18,19]$, we expected that some portion of AluS polymorphisms would reflect deletion polymorphisms, arising when AluS elements are (imperfectly) excised by an interstitial deletion. On the other hand, $A l u$ insertion polymorphisms classically arise by TPRT. Ongoing retrotransposition has resulted in thousands of $A l u$ insertion polymorphisms in the human genome, mostly confined to the AluY subfamilies [3]. Remarkably, we found that two of the four polymorphic AluS elements near GWAS signals described above are full-length elements that have all three defining features of a TPRT-mediated insertion [11]: (1) an intact 3' end, (2) a 3' poly-A tail, and (3) flanking TSDs. Therefore, these AluS polymorphisms appear to have arisen by TPRT.

To further expand the list of polymorphic AluS elements, we considered data from the most comprehensive effort to characterize structural variation in humans - the 1000 Genomes Project. In the most recent analysis there were 49 polymorphic AluS elements reported [2]. This list includes one of the AluS elements discussed above, thus bringing the total to 52 polymorphic AluS elements to characterize in more detail.

\section{Confirming AluS subfamily classification}

We first set out to confirm the AluS subfamily assignment of these 52 polymorphic elements using high stringency criteria (see Methods). Subfamily classification was performed using multiple established methods whenever possible. 
For the $49 \mathrm{AluS}$ polymorphisms that are annotated in the reference genome (hg19), we compared the subfamily calls made by RepeatMasker [20], the RepeatMasker track of the UCSC Genome Browser (hg19), and the 1000 Genomes Project [2], (Additional file 1: Table S1). In 22 cases there was complete agreement among these sources. In 22 cases there was minor disagreement, limited to a discrepancy in classification among AluS subfamilies. In the remaining five cases there was a more substantial disagreement regarding subfamily classification. In the first of the five cases, there was a discrepancy between subfamily classification by the 1000 Genomes Project as an AluSz element and subfamily classification by both RepeatMasker and the RepeatMasker track of the UCSC Genome Browser (hg19) as an $A l u J b$ element; this element was ultimately classified as an $A l u J$ element and excluded from later analysis. In the other four cases there was disagreement regarding the classification of the element in question as $A l u S$ versus $A l u Y$. To resolve this issue, we identified five diagnostic nucleotides that definitively distinguish between AluS and $A l u Y$ consensus sequences [21] when considering the six AluS subfamilies and the six most common $A l u Y$ subfamilies (Fig. 1a), [3]. The sequences of the four polymorphic $A l u$ elements were manually evaluated with respect to these positions. Three elements were severely $5^{\prime}$ truncated making subfamily classification difficult. In particular, two elements were so short that they did not contain the necessary diagnostic positions to determine $A l u Y$ versus $A l u S$ assignment, thus explaining the disagreement among the methods described above. Therefore, while these elements do have characteristics of classical TPRT insertions and may belong to the AluS subfamily, they were not included in our remaining analysis as they could not be confirmed to be AluS elements. The other element was less severely truncated than the two described above. It contained one diagnostic position distinguishing between AluS and AluY consensus sequences, which matched that of an AluY element, and was therefore also excluded from further analysis. The final element in question (located at 11q14.1) was full-length, which allowed for the evaluation of all five diagnostic positions between AluS and $A l u Y$ consensus sequences illustrated in Fig. 1a. The polymorphic sequence matched the AluS consensus sequence at the two diagnostic positions in the left monomer, the $A l u Y$ consensus sequence at two of the three diagnostic positions in the right monomer, and neither consensus sequence at the remaining nucleotide (Fig. 1b, Additional file 2: Figure S1). While such a chimeric element may have arisen by recombination between adjacent $A l u S$ and $A l u Y$ elements [3], the fact that this polymorphic element is flanked by identical TSDs makes this possibility unlikely. Given the full-length nature of this Alu polymorphism, we considered seven additional positions that largely, although not definitively, distinguish $A l u Y$ and AluS elements (Additional file 2: Figure S1). When considering all twelve positions, this element is consistent with only an $A l u S$ subfamily consensus sequence at six positions (highlighted in green) and consistent with only an $A l u Y$ subfamily consensus sequence at three positions (highlighted in red). At one position (highlighted in gray) this element is consistent with both AluS and AluY subfamily consensus sequences and at two positions (highlighted in yellow) it is consistent with neither AluS nor AluY subfamily consensus sequences; evaluation at these positions was, thus, uninformative. Due to predominating $A l u S$ features, the polymorphic $A l u$ element at 11q14.1 was ultimately classified as an AluS element and included in subsequent analysis.

Confirmation of subfamily classification of the three AluS polymorphisms not annotated in the reference genome was handled slightly differently. For two such AluS polymorphisms no subfamily assignment was made in the original report of the polymorphism; the element was only classified as belonging to the Alu family $[14,22]$ and subfamily assignment (to an AluS subfamily in both cases) was thus solely made using RepeatMasker [20]. For the third element, there was agreement between the original report [13] and RepeatMasker [20] with respect to subfamily assignment to the AluSg subfamily (Additional file 1: Table S1).

Further analysis thus focused on the $48 \mathrm{Alu}$ polymorphisms confirmed to be AluS elements using these high stringency criteria.

\section{AluS deletion polymorphism candidates and Non-classical Alu insertion candidates}

The overwhelming majority of previously reported polymorphic AluS elements in humans were classified as deletion polymorphisms $[2,13]$. However, based on our analysis of the AluS variants mapping near GWAS signals, it appears that some extant AluS polymorphisms have features of a TPRT-mediated Alu insertion event. Therefore, we set out to categorize the 48 polymorphic AluS elements as insertion or deletion polymorphisms.

Deletion polymorphisms may arise when fixed Alu elements are deleted through recombination, thus becoming polymorphic in the population. Such Alu deletions are often imprecise. While the pre-deletion allele contains the $A l u$ element, the post-deletion allele lacks the $A l u$ element, either in part or in its entirety, along with adjacent genomic sequence as well in some cases, depending on the recombination or end-joining event (Fig. 2). Thus, we defined deletion polymorphism candidates as polymorphisms that are not limited to the $\mathrm{Alu}$ element (i.e., due to the inclusion of adjacent genomic sequence) or that contain only a portion of the Alu 


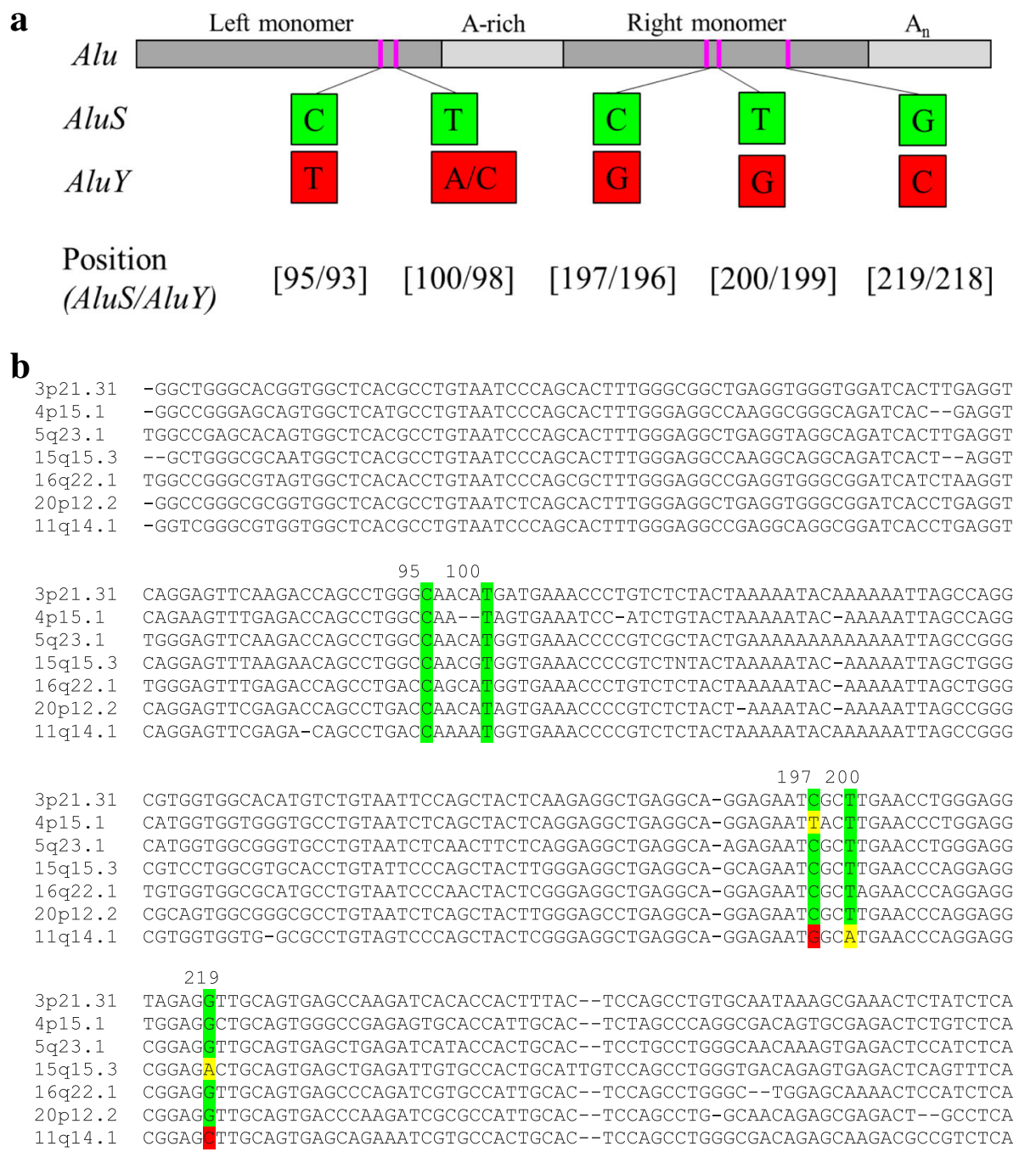

Fig. 1 Diagnostic nucleotides differentiate AluS and AluY elements. a Five diagnostic nucleotides that distinguish RepBase consensus sequences of all six AluS subfamilies (AluSc, AluSg, AluSp, AluSq, AluSx, AluSz,) from the most common AluY subfamilies (AluY, AluYa5, AluYa8, AluYb8, AluYb9, AluY (1) were identified [3]. b Five diagnostic positions indicated in part (a) in the context of Alu sequence confirms AluS subfamily classification. The seven AluS TPRT insertion candidates are shown. AluS specific nucleotides at the diagnostic positions are highlighted in green, AluY specific nucleotides at the diagnostic positions are highlighted in red, and nucleotides at the diagnostic positions that are neither AluS nor AluY specific are highlighted in yellow. Further analysis of the polymorphic Alu element at 11q14.1, which has features of both AluS and AluY elements, that led to its ultimate classification as an AluS element is shown in Additional file 2: Figure S1

element at that locus (i.e., only part of the $A l u$ is variably present among individuals and the rest of the Alu is fixed). Of the 48 polymorphic AluS elements, 39 were initially identified to be deletion polymorphism candidates based on this definition (Fig. 2).

We considered the possibility that some of these 39 deletion polymorphism candidates we identified may instead reflect non-classical Alu insertions (NCAI). While we were confident that the 33 cases in which the polymorphism does not include the entire $A l u$ element at that locus, so that part of the Alu is polymorphic and part is fixed (top five post-deletion allele categories in Fig. 2b), represent deletion polymorphisms, the six cases in which the polymorphism includes the entire Alu element as well as flanking genomic sequence (bottom two post-deletion allele categories in Fig. $2 \mathrm{~b}$ ) were evaluated more closely to determine if these could represent NCAI. These six polymorphisms could potentially be NCAI because over half of previously reported NCAI had $2 \mathrm{bp}$ to $2 \mathrm{~kb}$ of non-Alu sequence inserted along with the Alu fragment [23].

NCAI have several other characteristic features that we considered in evaluating these six polymorphisms. NCAI are typically $3^{\prime}$ truncated, thus lacking a poly-A tail, and also lack flanking TSDs [23]. Because they arise by an endonuclease-independent (ENi) mechanism, no 


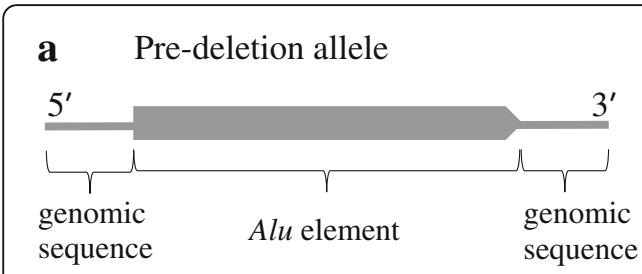

b Observed post-deletion alleles

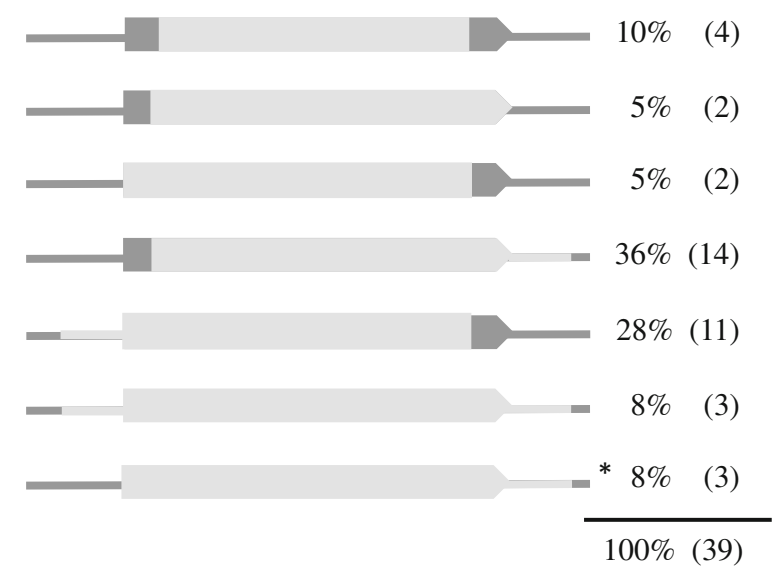

Fig. 2 Characterization of the 39 AluS deletion polymorphism candidates. a The pre-deletion allele contains the Alu element (dark gray block arrow); flanking genomic sequence is depicted as a thin dark gray line on both sides of the Alu element. b Schematic of the seven categories of post-deletion alleles observed among the 39 AluS deletion polymorphism candidates. Polymorphic sequences are depicted in light gray; the dark gray parts indicate the sequences that do not vary among individuals. Deletion polymorphism candidates are defined as polymorphisms that encompass only a portion of the Alu element at that locus (e.g. the top five post-deletion allele categories) or that are not limited to only the Alu element (e.g. the bottom four categories). The last category (marked by an asterisk) includes two elements that have features of non-classical Alu insertions and may not be true deletion polymorphisms (see Results). All Alu elements are shown 5' to 3'. Observed frequencies of each post-deletion allele category among the 39 AluS deletion polymorphism candidates are shown

L1 ORF2p cleavage sites are observed at the insertion site [23]. Previous studies found that most NCAI are also associated with deletions at the insertion site ranging from $1 \mathrm{bp}$ to $\sim \mathrm{kb}[11,23-25]$.

The three polymorphisms that include both $5^{\prime}$ and $3^{\prime}$ flanking genomic sequence in addition to the AluS element were all confirmed to be deletion polymorphisms. All three AluS elements included in these polymorphisms are full-length with $3^{\prime}$ poly-A tails, and are flanked by identical TSDs ranging from 11 to $17 \mathrm{bp}$; these features exclude the possibility that these are NCAI [23]. We next considered the three polymorphisms that include 3' flanking genomic sequence in addition to the AluS element in its entirety. One of these was confirmed to be a deletion polymorphism, by virtue of being a full-length AluSz element with a 3' poly-A tail; flanking TSDs could not be identified due to the presence of another Alu insertion immediately $5^{\prime}$ of this element. The two remaining polymorphisms in this category appear to be NCAI (Fig. 3, Additional file 3: Table S2). Both of these elements are Alu fragments, truncated at both the $5^{\prime}$ and $3^{\prime}$ ends, one with 95 bp of homology to the left monomer and A-rich region of the AluSc consensus sequence and the other with $77 \mathrm{bp}$ of homology to the right monomer of the AluSq2 consensus sequence. Flanking TSDs are not observed in either case. No previously reported L1 ORF2p endonuclease cleavage sites [7] are present at either insertion site, consistent with ENi insertion [23]. Both polymorphisms include short stretches of non-Alu sequence at the 3' end (11 bp with the AluSc fragment and 21 bp with the AluSq2 fragment); the polymorphism that includes the AluSq2 fragment is also associated with a 14 bp deletion at the insertion site. These features are thus consistent with those previously reported for NCAI [23]. Both of these NCAI candidates were PCR validated to be polymorphic in the population (Additional file 3: Table S2).

In summary, of the 48 polymorphic AluS elements, $77 \%(37 / 48)$ are deletion polymorphism candidates (Additional file 4: Table S3) and 4\% (2/48) appear to be NCAI. The remaining nine insertion polymorphism candidates were next evaluated in more detail.

\section{Polymorphic AluS insertion likely arising by internal priming}

One AluS insertion polymorphism candidate, which was PCR validated to be polymorphic in the population (Additional file 3: Table S2), does not have all of the defining characteristics of retrotransposon insertions occurring by TPRT [11]. The polymorphic AluSq2 element at $8 \mathrm{p} 11.23$ is full-length and flanked by TSDs but completely lacks a 3' poly-A tail (Fig. 3, Additional file 3: Table S2). Thus, it likely arose through an insertion mechanism other than TPRT. Specifically, its features are characteristic of an element that inserted by internal priming [26]. While in classic TPRT, reverse transcription begins at the $3^{\prime}$ end of the poly-A tail [27], in this case reverse transcription likely began at the $5^{\prime}$ end of the poly-A tail, thus accounting for the insertion of a full-length element, only lacking the poly-A tail. While poly-A tail length tends to decrease over time after insertion toward a more stable equilibrium value, the polyA tail is unlikely to be completely eliminated and no such cases have been reported even among older Alu subfamilies [27]. Thus, insertion of this tail-less polymorphic AluSq2 element most likely occurred by internal priming. Absence of an L1 ORF2p endonuclease cleavage site at the insertion site of this element is consistent with the fact that the internal priming 


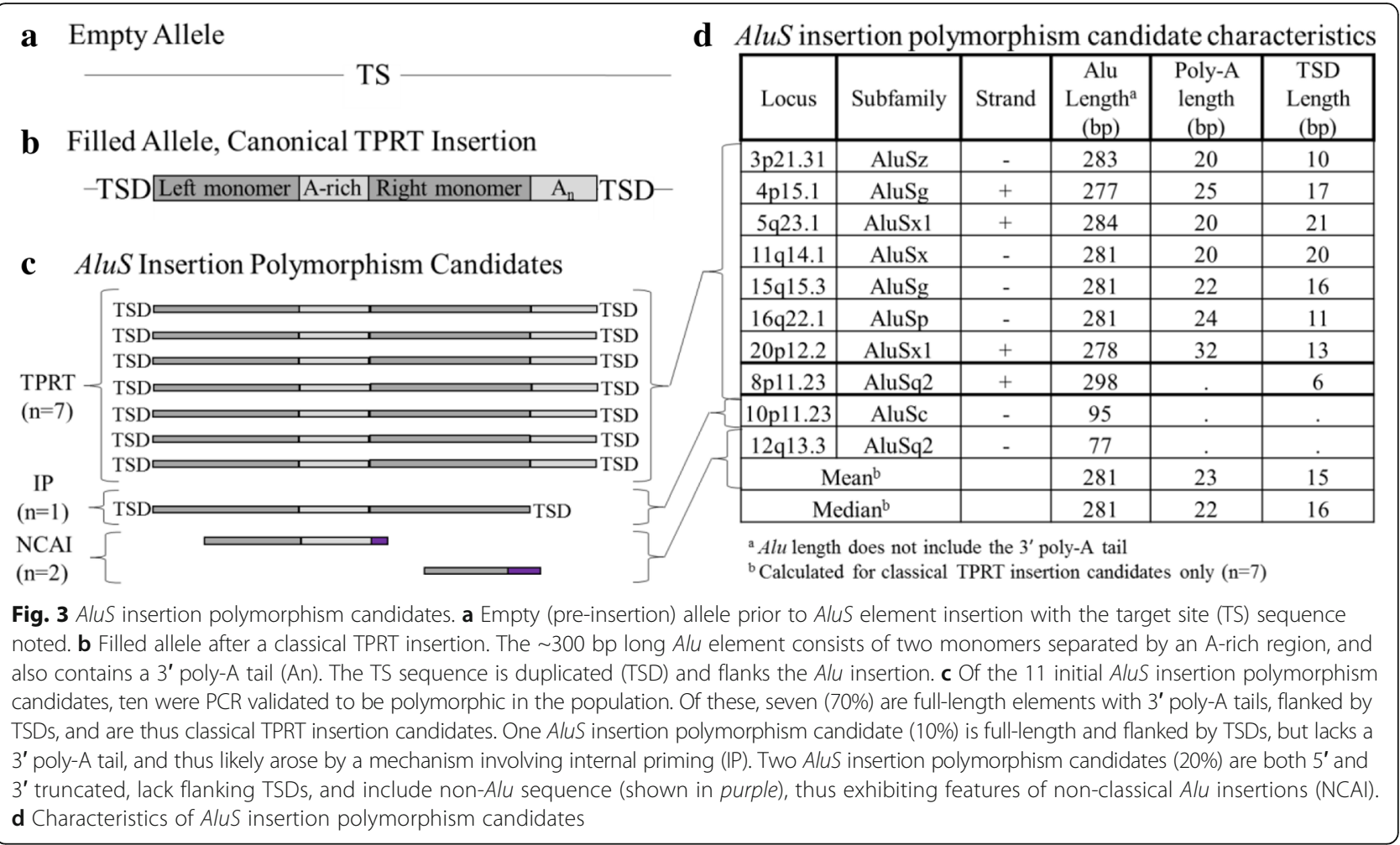

mechanism of insertion does not always rely on L1 ORF2p endonuclease and may occur at the site of staggered double-strand breaks (DSBs), thus creating flanking TSDs [26].

\section{Polymorphic AluS insertions likely arising by target-primed reverse transcription}

Eight AluS polymorphisms have all the defining features of retrotransposon insertions that have arisen by TPRT [11]). However, we were only able to PCR validate seven of them to be polymorphic in the population (Additional file 3: Table S2). Therefore, further analysis only focused on the seven validated AluS TPRT insertion candidates (Figs. 1b and 3).

These seven AluS elements are full-length insertions (277-284 bp), with intact 3' poly-A tails (20-32 bp), and are flanked by TSDs (10-21 bp) (Fig. 3, Additional file 3: Table S2). To evaluate further the possibility of TPRTmediated insertion, we searched for the L1 ORF2p endonuclease cleavage site at the insertion site of each of the seven elements (Fig. 4, Additional file 3: Table S2). The endonuclease cleavage sites for the seven loci fall within the distribution previously reported by Konkel et al. [7] (Fig. 4, Additional file 3: Table S2). Thus, we see features consistent with TPRT-mediated insertion in seven polymorphic AluS elements of 48 total AluS polymorphisms evaluated in this report (15\%).
Percent divergence from subfamily consensus sequence and estimated Age of TPRT insertion candidates

These seven AluS insertion candidates could reflect fairly recent TPRT-mediated insertions, or could be old insertions slow to reach fixation in human populations. To consider the age of these sequences relative to other AluS elements, we compared them to their respective subfamily consensus sequences. Relatively new insertions would not have had much time to accumulate random mutations (i.e., neutral substitutions) and would conserve many of the features of $A l u$ elements required for retrotransposition.

We evaluated the degree of divergence of each AluS TPRT insertion candidate from its subfamily consensus sequence, and found that the divergence ranges from 5.2

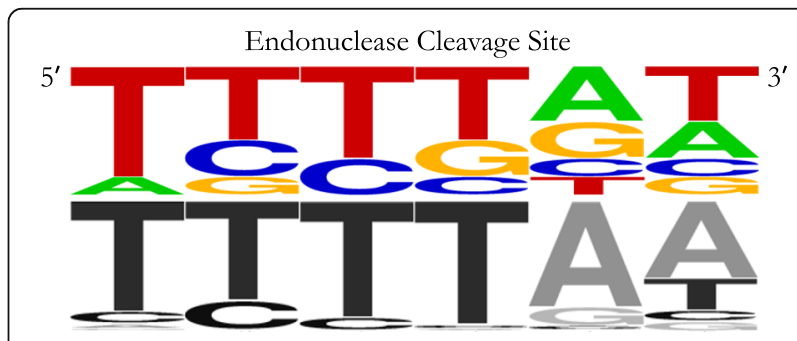

Fig. 4 L1 ORF2p endonuclease cleavage sites for all classical TPRT insertion candidates $(n=7)$ displayed as a WebLogo diagram [47]. The negative strand is depicted, $5^{\prime}$ to $3^{\prime}$. The published consensus sequence is depicted below in grayscale [7] 
to $11.2 \%$, with a mean of $8.9 \%$ and a median of $10.2 \%$ (Fig. 5a). We found that these seven TPRT insertion candidates are significantly less diverged from their respective subfamily consensus sequence than are all the AluS elements annotated in the reference genome $(n=686,955)$ from their respective subfamily consensus sequence ( $p=0.0038$, permutation test), (Fig. 5b-c). This supports the hypothesis that these insertions likely occurred more recently than at the peak AluS activity approximately 35-60 million years ago $[18,19]$.

We also estimated the age of the TPRT insertion candidates using previously reported substitution rates at $\mathrm{CpG}$ and non $\mathrm{CpG}$ sites. Since $\mathrm{Alu}$ elements are rich in CpG sites, which are known to have an appreciably higher mutation rate than non-CpG sites [28], CpG and non-CpG based age estimates are both valuable metrics in addition to percent divergence from the consensus sequence. Age estimates were calculated for each TPRT insertion candidate as previously described [28-32] (see Methods). CpG based age estimates range from 13.9 to 48.6 million years and non-CpG based age estimates range from 22.7 to 37.5 million years (Fig. 5a). Despite the range of estimated ages among the TPRT insertion candidates, overall they appear to have inserted more recently than at the peak of AluS activity 35-60 million years ago $[18,19]$.

\section{Conservation of functionally significant Alu sequence features in TPRT insertion candidates}

To evaluate the degree of conservation of functionally significant $A l u$ sequence features, we focused on three specific regions - the RNA polymerase III promoter A and B boxes within the left monomer [33], the SRP9/14 major and minor binding sites within the left and right monomers [16], and AC dinucleotides within the left and right monomers previously reported to be important for maintaining Alu RNA secondary structure [34]. Since these sequences are critical for successful retrotransposition, they would presumably be present and functional in the source element templating each TPRT insertion variant, and would be highly conserved in recent insertions.

The RNA polymerase III promoter, which is important for efficient transcription [33], is indeed well conserved (Fig. 6a, Additional file 5: Figure S2). Nine of the $11 \mathrm{nu}$ cleotides in the A box consensus sequence are fully conserved in all seven AluS elements, with infrequent departures from the consensus at the other two positions; five AluS elements have an A box that exactly a

\begin{tabular}{|c|c|c|c|c|}
\hline Locus & Subfamily & \% Divergence & $\begin{array}{c}\text { CpG Based Age } \\
\text { (myrs) }\end{array}$ & $\begin{array}{c}\text { Non-CpG Based Age } \\
\text { (myrs) }\end{array}$ \\
\hline $3 \mathrm{p} 21.31$ & $A l u S z$ & 11.2 & 48.6 & 34.0 \\
\hline $4 \mathrm{p} 15.1$ & $A l u S g$ & 10.9 & 37.8 & 37.5 \\
\hline $5 \mathrm{q} 23.1$ & $A l u S x 1$ & 10.3 & 41.7 & 36.8 \\
\hline $11 \mathrm{q} 14.1$ & $A l u S x$ & 5.7 & 13.9 & 22.7 \\
\hline $15 \mathrm{q} 15.3$ & $A l u S g$ & 10.2 & 37.8 & 31.7 \\
\hline $16 \mathrm{q} 22.1$ & $A l u S p$ & 8.9 & 33.8 & 25.2 \\
\hline $20 \mathrm{p} 12.2$ & $A l u S x 1$ & 5.2 & 13.9 & 28.3 \\
\hline \multicolumn{2}{|c|}{ Mean } & 8.9 & 32.5 & 30.9 \\
\hline \multicolumn{2}{|c|}{ Median } & 10.2 & 37.8 & 31.7 \\
\hline
\end{tabular}

b

Percent Divergence from AluS Subfamily

Consensus Sequence

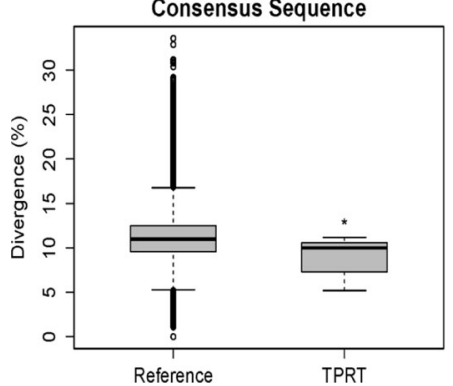

c Percent Divergence

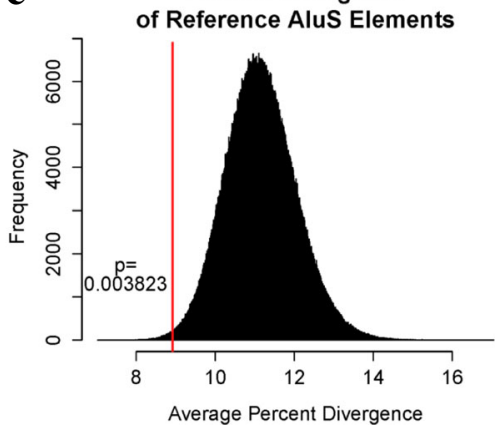

Fig. 5 Estimated age and degree of divergence from subfamily consensus sequence of AluS TPRT insertion candidates. a For each of the AluS TPRT insertion candidates $(n=7)$ percent divergence from the respective AluS subfamily consensus sequence is shown along with estimated ages for the elements based on $\mathrm{CpG}$ and non- $\mathrm{CpG}$ substitution rates. b Boxplot of percent divergence from the respective AluS subfamily consensus sequence of all AluS elements annotated in the reference genome $(n=686,955)$ and the TPRT insertion candidates $(n=7)$. c The TPRT insertion candidates $(n=7)$ are significantly less diverged from their respective AluS subfamily consensus sequence than are all the AluS elements annotated in the reference genome $(n=686,955)$ from their respective subfamily consensus sequence $(p=0.0038$, permutation test). The distribution of the mean percent divergence of $1 \times 10^{6}$ random samples of $n=7$ drawn from the total 686,955 AluS elements annotated in the reference genome is shown. The mean percent divergence of the seven TPRT insertion candidates is shown as a vertical red line 
$\mathbf{a}$

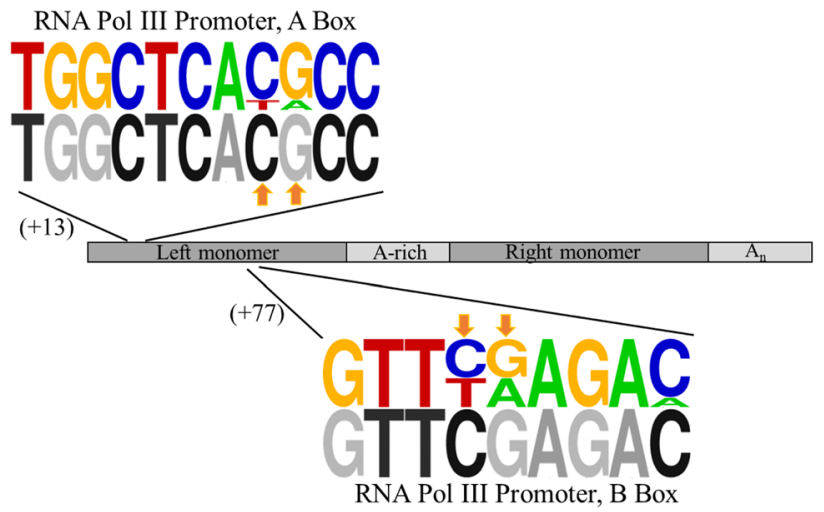

b

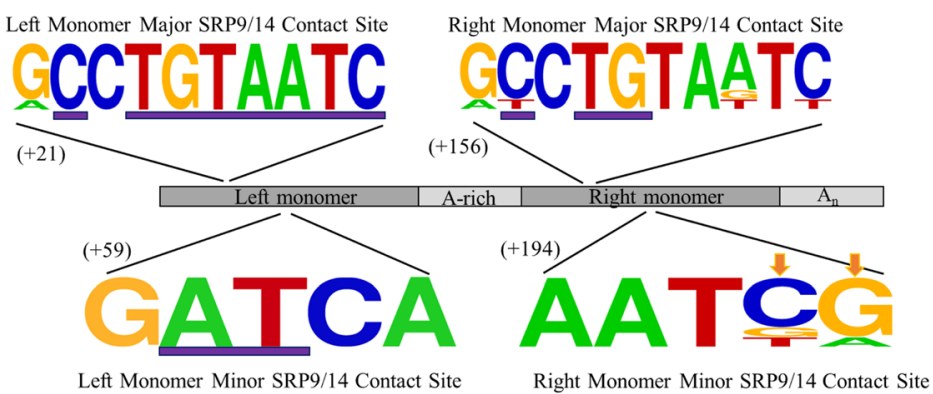

c

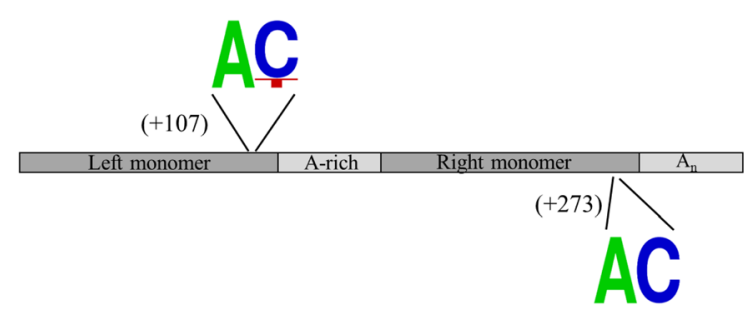

Fig. 6 Conservation of functionally significant Alu sequence features in the seven classical TPRT insertion candidates. a WebLogo diagram [47] of the RNA polymerase III promoter A and B boxes, with the published consensus sequences depicted below in grayscale [33]. CpG sites are indicated by an orange arrow. $\mathbf{b}$ WebLogo diagram [47] of the SRP9/14 binding sites. Previously reported most highly conserved sites within the SRP9/14 binding sites of elements capable of retrotransposition are underlined by a purple bar [16]. CpG sites are indicated by an orange arrow. c WebLogo diagram [47] of two AC dinucleotides in the Alu sequence reported to play a critical role in maintaining the closed loop conformation of Alu RNA that is important for interaction with SRP9/14 and efficient retrotransposition [34]

matches the consensus sequence, and the remaining two elements have one mismatch, each at a different position. Six of the nine nucleotides in the B box consensus sequence are fully conserved in all seven AluS elements, with some variation at the other three positions; two AluS elements have a B box that exactly matches the consensus sequence, four elements have one mismatch, and one element has three mismatches. Two AluS elements have both an A and a B box that exactly matches the consensus sequence. Across the A and B boxes, four of the five imperfectly conserved positions are at CpG sites, which are known to have a higher mutation rate due to spontaneous deamination of methylated cytosines at these positions [28].

The SRP9/14 binding sites, which, as predicted by the ribosome-binding model, are important for interaction with SRP9/14, and subsequently with the ribosome and L1 retrotransposition machinery [16, 35] are also highly conserved (Fig. 6b, Additional file 5: Figure S2). In the left monomer, all of the previously reported most highly conserved nucleotides in the major and minor binding sites of elements capable of retrotransposition were fully conserved in all seven AluS elements. In the right monomer, two of the three nucleotides previously reported to be most highly conserved in the major binding site of elements capable of retrotransposition were fully conserved in all seven AluS elements, and the third nucleotide was conserved in six of the seven AluS elements. In all, six of the seven AluS elements have full conservation of all the nucleotides previously reported to be most highly conserved within the SRP9/14 binding sites of elements capable of retrotransposition. 
Finally, there was strong conservation of both the left and right monomer $\mathrm{AC}$ dinucleotides (Fig. 6c, Additional file 5: Figure S2), [34]. These positions have been reported to play a critical role in stabilizing the closed loop conformation of Alu RNA that is important for interaction with SRP9/14 via the SRP9/14 binding sites [34] thus lending further support to the ribosome-binding model [35]. The left monomer AC dinucleotide was largely conserved among the seven AluS TPRT insertion candidates; there was no variation at the A position, and variation at the $\mathrm{C}$ position in only one of the seven AluS TPRT insertion candidates. There was perfect conservation of the right monomer AC dinucleotide in all seven AluS elements.

\section{Discussion}

In this report, we annotate 52 previously reported polymorphic AluS elements, confirm that 48 of them do indeed belong to the AluS subfamily using high stringency criteria, and comment on their likely mechanism of origin. While most of these appear to be deletion polymorphisms consistent with previous reports [2, 13], we present evidence that seven of these polymorphic AluS elements have features consistent with insertion by TPRT. This implies that some AluS elements may have been more active recently than previously thought $[7,19]$ and expands the significance of AluS retrotransposition in contributing to structural variation in the human genome.

The overwhelming majority of AluS insertions in the human genome are fixed, consistent with the fact that the AluS subfamily was most active 35-60 million years ago $[18,19]$. Based on this, most AluS elements that are polymorphic among humans are expected to be deletion polymorphisms. Deletion events are highly unlikely to include only the Alu element in its entirety; while a recombination event between the flanking TSDs would yield a precise deletion of the intervening Alu element, such events are extremely rare [36]. Thus, we defined a deletion polymorphism candidate as one that was not limited to only the Alu element or did not contain the Alu element at that locus in its entirety. As expected, the majority $(77 \%, 37 / 48)$ of polymorphic AluS elements evaluated in this study are deletion polymorphism candidates.

The remaining 23\% (11/48) of AluS polymorphisms characterized in this report are insertion polymorphism candidates, of which ten were PCR validated to be polymorphic in the population. Three mechanisms of Alu insertion have been previously reported - TPRT [4], internal priming (IP) [26], and NCAI/ENi [23]- and are all represented among the ten AluS insertion polymorphism candidates (Fig. 3). Two insertion polymorphisms have features of NCAI [23]. The AluS elements included in these polymorphisms are both $5^{\prime}$ and $3^{\prime}$ truncated and lack flanking TSDs; no previously reported L1 ORF2p endonuclease cleavage sites could be identified at the insertion site. Both polymorphisms include non- $A l u$ sequence at the $3^{\prime}$ end and one polymorphism is associated with a deletion at the insertion site. These are all characteristic features of ENi insertion, a mechanism in which Alu transcripts are utilized by the cell to repair DSBs, thus leading to insertion polymorphisms [23]. One AluS insertion polymorphism appears to have arisen by a mechanism involving IP [26]. While this element is full-length and flanked by TSDs, it lacks a 3' poly-A tail and no L1 ORF2p endonuclease cleavage site could be identified at the insertion site. These features are consistent with insertion by IP, which may be an alternative mechanism to repair staggered DSBs [26].

The majority of AluS insertion polymorphism candidates $(70 \%, 7 / 10)$ have all the features of classical TPRT insertions, which may suggest that AluS elements were more active recently than previously thought. Our observation is consistent with the "stealth driver" model, which posits that some subfamily members retain low levels of activity over tens of millions of years, long after the subfamily's peak activity [37]. The seven TPRT insertion candidates do not appear to all be products of a single persistent "stealth-driver" element, however. These elements belong to four different subfamilies (three AluSx elements, two AluSg elements, one AluSp element, and one $A l u S z$ element), which strongly implies that multiple source elements contributed to these insertions.

The ability of AluS elements to retrotranspose in humans is considered minimal due to the fact that few polymorphic insertions with features consistent with TPRT-mediated insertion have been identified [7]. However, a de novo AluSq/Sp insertion in an exon of BRCA1 with features of TPRT-mediated insertion has been reported, thus suggesting that some AluS elements may still be retrotransposition competent and may, moreover, influence disease risk [38]. Furthermore, AluS consensus sequences and some genomic AluS elements have been shown to be active in in vitro retrotransposition assays [16]. Importantly, those elements diverging more than $10 \%$ from their consensus sequence are inactive, thus highlighting the importance of Alu sequence integrity for retrotransposition capability [16]. If the seven candidate TPRT insertions we evaluated did indeed occur fairly recently, then the sequences would not have had much time to accumulate random mutations and would not yet significantly diverge from their respective subfamily consensus sequences. This is indeed what we observe, and the seven TPRT insertion candidates, with a mean percent divergence of $8.9 \%$, are significantly less diverged from their respective subfamily consensus 
sequence than are all the AluS elements annotated in the reference genome from their respective subfamily consensus sequence ( $p=0.0038$, permutation test).

Similarly, specific sequence features known to be functionally important for retrotransposition, namely the RNA polymerase III promoter, SRP9/14 binding sites, and AC dinucleotides involved in maintaining Alu RNA secondary structure, are highly conserved. The RNA polymerase III promoter is important for efficient transcription of the element [33], and the AC dinucleotides play a critical role in maintaining the closed loop conformation of Alu RNA that is important for interaction with SRP9/14 via the SRP9/14 binding sites, which thus allows the Alu transcript to associate with the ribosome and ultimately positions it in close proximity to the $\mathrm{L} 1$ encoded proteins required for retrotransposition [16, 34, 35, 39, 40]. The high overall level of conservation suggests these seven TPRT candidates have not diverged significantly from elements that would be capable of retrotransposition, consistent with a recent insertion templated by a TPRT competent element.

While polymorphic AluS elements have previously been reported, most discovery efforts aimed to identify only young Alu subfamilies and did not include AluS subfamily consensus sequences in their algorithms $[2,12,41]$. The AluS polymorphisms reported by the 1000 Genomes Project (phase 3) [2] were identified using a pipeline for mapping insertion/deletion polymorphisms rather than the Mobile Element Locator Tool; thus, their identification of polymorphic AluS elements was limited to those that are in the reference genome. Notably, a recent study by Hormozdiari et al. [13] that did intend to identify polymorphic AluS elements, reports that $9.4 \%$ of identified polymorphic $\mathrm{Alu}$ elements belong to the AluS subfamily. However, the authors inferred that the majority of these were likely deletion polymorphisms or insertions arising by endonucleaseindependent mechanisms as opposed to novel TPRT events [13]. Here, we present evidence that while, in agreement with previous studies, the majority $(77 \%, 37 / 48)$ of the polymorphic AluS elements evaluated in this report are likely deletion polymorphisms, 15\% (7/48) have features of TPRT-mediated insertions. While these may represent insertions that occurred at the peak of AluS activity and have by some means been slow to progress to fixation in the population, the strong conservation of sequence features important for retrotransposition (e.g. RNA polymerase III promoter, SRP9/14 binding sites) implies that some of these may be fairly recent insertions. As most previous discovery efforts did not specifically target identification of AluS polymorphisms [2, 12, 41], the majority of the polymorphic AluS TPRT candidates described here were identified because there were no reads mapping to the annotated Alu element in the reference genome. Therefore, there is a potential for more polymorphic AluS elements, especially less common variants not already annotated in the reference genome, to be identified with targeted discovery efforts.

\section{Conclusions}

In summary, we present evidence of polymorphic AluS elements in the human genome with features consistent with TPRT-mediated insertion events. These findings imply that multiple AluS subfamilies may have been more active recently than previously thought, consistent with the "stealth driver" model [37]. Our analysis also substantiates the concept that AluS element deletions are an important contributor to structural variation in humans. In fact, since most methodologies for finding insertion variants have focused on identifying polymorphic AluY elements, we expect there to be more yet uncharacterized polymorphic AluS insertions and deletions in the human genome. Our findings stress the importance of future structural variant discovery efforts to identify polymorphic AluS elements.

\section{Methods \\ Cataloging polymorphic Alu elements}

We compiled a list of previously reported polymorphic Alu elements [12-14, 22, 41-44], totaling 13,572 Alu variants across the human genome. To focus on $A l u$ variants with potential functional consequences, we selected polymorphic Alu elements within linkage disequilibrium (LD) blocks $\left(r^{2} \geq 0.8\right)$ around GWAS trait-associated SNPs (TAS) with $p<10^{-9}$ from the NHGRI-EBI GWAS catalog [45] and Sanger sequenced 112 of them. We used RepeatMasker [20] to make subfamily assignments [17].

We also considered the list of polymorphic AluS elements reported by the 1000 Genomes Project, phase 3 [2]. In this report [2], the Alu polymorphisms of interest were identified using deletion discovery algorithms, and then classified as AluS using the AluScan algorithm as part of the Mobile Element Locator Tool (MELT).

\section{Classifying polymorphic Alu elements by subfamily}

Subfamily assignment of the $52 \mathrm{AluS}$ polymorphisms ascertained for this study was confirmed using high stringency criteria, using multiple established methods when possible. Subfamily assignments for all 52 elements were made using RepeatMasker [20].

For the 49 elements annotated in the reference genome, subfamily assignments from RepeatMasker [20], the UCSC Genome Browser RepeatMasker track (hg19), and the 1000 Genomes Project (obtained using the AluScan algorithm as part of the Mobile Element Locator Tool (MELT)) [2] were compared (Additional file 1: Table S1). When there was disagreement in classification 
among AluS subfamilies, the final assignment was made in accordance with RepeatMasker [20], both because it is a well trusted tool and for the sake of consistency, since it is the main tool available to classify the three non-reference genome polymorphic AluS elements presented in this report. When there was more substantial disagreement with respect to $A l u S$ versus $A l u Y$ assignment, the elements were manually classified. RepBase consensus sequences [21] for the following subfamilies were obtained (AluSc, AluSg, AluSp, AluSq, AluSx, AluSz, AluY, AluYa5, AluYa8, AluYb8, AluYb9, AluYc1) and aligned using the MUSCLE multiple sequence alignment tool [46], (Additional file 2: Figure S1). Five diagnostic nucleotides that distinguish all six AluS subfamilies from the six AluY subfamilies included in the alignment were identified (Fig. 1a), [3]. The polymorphic Alu sequences were evaluated at these positions and the final assignment was made based on which consensus sequence the element more closely resembled.

For the three elements not annotated in the reference genome, the RepeatMasker [20] classification was compared to the subfamily classification indicated in the original report of the polymorphism, if available (Additional file 1: Table S1). When no subfamily classification was made in the original report, subfamily assignment was made solely using RepeatMasker.

\section{Annotating reference genome polymorphic Alu elements} Forty-five of the 48 polymorphic Alu elements confirmed to belong to the AluS subfamily are in the reference genome (hr19). To confirm the extent of the polymorphism, we compared the annotated polymorphic sequence [2] to the reference genome (hg19) and the RepeatMasker track (UCSC Genome Browser, hg19). Deletion polymorphisms were initially identified as those not limited to only the Alu element (i.e., due to the inclusion of adjacent genomic sequence) or those that did not contain the Alu element at that locus in its entirety (i.e., part of the $A l u$ is polymorphic among individuals and the rest of the Alu is present in everyone). Deletion polymorphism candidates that included the entire Alu element as well as additional 5' and/or 3' flanking genomic sequence were further investigated to determine whether they could represent NCAI; this was done by evaluating the Alu element included in the polymorphism for degree of truncation, presence of a $3^{\prime}$ poly-A tail, and flanking TSDs. Further analysis focused only on the insertion polymorphism candidates. When present, the 3' poly-A tail was identified. Flanking genomic sequence was obtained from the reference genome and used to manually identify TSDs; TSD length was maximized at the expense of poly-A tail length when applicable.

\section{Annotating non-reference genome polymorphic Alu elements}

Three of the 48 polymorphic Alu elements confirmed to belong to the AluS subfamily are not in the reference genome (hg19). These loci were PCR amplified with primers flanking the insertion site from individuals from the Centre d'Etude du Polymorphisme Humain (CEPH) from Utah (CEU) HapMap Reference panel to obtain sequences for the filled and empty alleles with respect to each Alu element. These PCR products were then cloned and Sanger sequenced. The resulting sequences of the filled and empty alleles were aligned to each other and the reference genome (hg19) to determine the extent of the polymorphism, including the position of the breakpoints with respect to the Alu sequence. The identified polymorphic sequence was then analyzed by RepeatMasker [20] to determine which parts of the polymorphic sequence had Alu homology and make a subfamily assignment. When present, the 3 ' poly-A tail and TSDs were manually identified and annotated as described in the section above.

\section{PCR validation of insertion polymorphism candidates}

Primers flanking the insertion site of the 11 insertion polymorphism candidate elements were designed, the regions were PCR amplified, and the PCR amplicons were resolved using gel electrophoresis. Polymorphic AluS elements were confirmed by amplification of two different sized alleles from the locus, that, when viewed on the agarose gel, differ in size corresponding to the size of the respective AluS element. Loci at which two alleles, the pre-insertion allele and AluS-containing allele, could be observed were validated polymorphisms. Additional file 3: Table S2 includes the DNA samples and PCR primer sequences used for validation of each locus.

\section{Percent divergence from AluS subfamily consensus sequence}

For the TPRT insertion candidates classified as AluS elements by RepeatMasker [20] $(n=6)$, the percent divergence from the respective subfamily consensus sequence was obtained from that analysis. For the $A l u$ element at 11q14.1, the percent divergence from the RepBase AluSx consensus sequence [21] was determined using the MUSCLE multiple sequence alignment tool [46]. To extend analysis to all AluS elements the genome, we used the Table Browser function in the UCSC Genome Browser to obtain for all AluS elements annotated in the RepeatMasker track (hg19) the percent divergence from the respective AluS subfamily consensus sequence. A permutation test was performed using $\mathrm{R}$ version 3.2.3 to determine whether the mean percent divergence of the seven TPRT insertion candidates 
$(\bar{x}=8.914) \quad$ was significantly lower than the mean percent divergence of all AluS elements in the reference genome. From the total 686,955 AluS elements annotated in the reference genome, $1 \times 10^{6}$ random samples of $n=7$ were drawn with replacement, and the mean percent divergence of each sample was calculated to obtain a distribution of the means (X), (Fig. $5 \mathrm{c})$. The $p$ value $(p=0.003823)$ was calculated as the fraction of random samples with means less than the observed percent divergence of the seven TPRT insertion candidates $(\operatorname{Pr}(X<\bar{x}))$.

\section{Alu element age estimates}

Two estimates for the age of the Alu elements were calculated based on CpG and non-CpG substitution rates as previously reported [28-32]. Briefly, AluS TPRT insertion candidate sequences (without the poly-A tail) were aligned to the respective AluS subfamily RepBase consensus sequence [21] using the MUSCLE multiple sequence alignment tool [46]. The number of substitutions at $\mathrm{CpG}$ and non-CpG sites were counted; for the CpG sites, only $\mathrm{C}$ to $\mathrm{T}$ and $\mathrm{G}$ to $\mathrm{A}$ substitutions were counted. The substitution densities were then calculated by dividing the number of observed $\mathrm{CpG}$ (or non-CpG) substitutions by the total number of $\mathrm{CpG}$ (or non-CpG) sites in the consensus sequence. The age of these elements was then calculated using a neutral rate of evolution of $\mathrm{k}=1.5 \times 10^{-9}$ per nucleotide position per year for the non-CpG sites [31] and a six-fold higher rate of evolution $\left(\mathrm{k}=9 \times 10^{-9}\right.$ per nucleotide position per year) for the CpG sites as determined by Xing et al. [28].

\section{Additional files}

Additional file 1: Table S1. Comparison of subfamily classification of A/u polymorphisms $(n=52)$ using multiple established methods. (XLSX $14 \mathrm{~kb})$

Additional file 2: Figure S1. Diagnostic nucleotides differentiate AluS and AluY subfamily consensus sequences. a. To identify diagnostic nucleotides differentiating AluS and AluY subfamily consensus sequences, RepBase consensus sequences of all six AluS subfamilies (AluSc, AluSg, AluSp, AluSq, AluSX, AluSz,) and the most common AluY subfamilies (AluY, AluYa5, AluYa8, AluYb8, AluYb9, AluYc1) were aligned. Five diagnostic nucleotides that distinguish all six AluS subfamilies from six AluY subfamilies included in the alignment were identified (highlighted in magenta). Seven additional positions that largely, but not definitively, distinguish between AluS and AluY elements are also illustrated (highlighted in cyan). b. Full-length polymorphic Alu element at 11q14.1 that has features of both AluS and AluY elements. Manual evaluation at the 12 diagnostic nucleotides that differentiate AluS and AluY elements led to its final classification as an AluS element due to predominating AluS features. This element is consistent with only an AluS subfamily consensus sequence at six positions (highlighted in green) and consistent with only an AluY subfamily consensus sequence at three positions (highlighted in red). At one position (highlighted in gray) this element is consistent with both AluS and AluY subfamily consensus sequences and at two positions (highlighted in yellow) it is consistent with neither AluS nor AluY subfamily consensus sequences; evaluation at these positions was, thus, uninformative. (PDF $72 \mathrm{~kb}$ )
Additional file 3: Table S2. Polymorphic AluS insertion candidates $(n=11)$. (XLSX $17 \mathrm{~kb})$

Additional file 4: Table S3. Sequences of deletion and Alu-containing alleles of AluS deletion polymorphisms ( $n=37)$ [2]. (XLSX $16 \mathrm{~kb})$

Additional file 5: Figure S2. Functionally significant Alu sequence features annotated in TPRT insertion candidates $(n=7)$. (PDF $203 \mathrm{~kb}$ )

\section{Abbreviations}

CEPH: Centre d'Etude du Polymorphisme Humain; CEU: Centre d'Etude du Polymorphisme Humain (CEPH) from Utah; DSB: Double-strand break; ENi: Endonuclease-independent; GWAS: Genome-wide association study; IP: Internal priming; LD: Linkage disequilibrium; LINE-1, L1: Long INterspersed Element-1; MELT: Mobile Element Locator Tool; NCAI: Nonclassical Alu insertion; NHGRI-EBI: National Human Genome Research Institute - European Bioinformatics Institute; ORF2p: Open reading frame 2 protein; SNP: Single nucleotide polymorphism; SRP: Signal recognition particle; TAS: Trait-associated SNP; TPRT: Target-primed reverse transcription; TSD: Target site duplication

\section{Acknowledgements}

We thank Daniel Ardeljan for helping with the statistical analysis and for critically reading the manuscript.

\section{Funding}

This work was supported by US National Institute of Health awards R01CA163705 and R01GM103999 (KHB), and the Howard Hughes Medical Institute (HHMI) Medical Research Fellows Program (MSK).

\section{Availability of data and materials}

All data analyzed in this study are either included in this published article (and its supplementary information files) or may be accessed from the published article and supplementary files of: Sudmant PH, Rausch T, Gardner EJ, Handsaker RE, Abyzov A, Huddleston J, Zhang Y, Ye K, Jun $\mathrm{G}$, Fritz MHY et al. An integrated map of structural variation in 2,504 human genomes. Nature. 2015; 526:75-81 (http://dx.doi.org/10.1038/ nature15394), [2]

\section{Authors' contributions}

MSK annotated and analyzed sequences, and wrote the manuscript. JPS conducted molecular biology experiments and annotated sequences. KHB and LMP conceived of the study, oversaw the project, and critically revised the manuscript. All authors read and approved the final manuscript.

\section{Competing interests}

The authors declare that they have no competing interests.

\section{Consent for publication}

Not applicable.

Ethics approval and consent to participate Not applicable.

\section{Publisher's Note}

Springer Nature remains neutral with regard to jurisdictional claims in published maps and institutional affiliations.

\section{Author details}

'Department of Pathology, Johns Hopkins University School of Medicine, Miller Research Building (MRB) Room 447, 733 North Broadway, Baltimore, MD 21205, USA. ${ }^{2}$ McKusick-Nathans Institute of Genetic Medicine, Johns Hopkins University School of Medicine, Miller Research Building (MRB) Room 447, 733 North Broadway, Baltimore, MD 21205, USA. 
Received: 18 January 2017 Accepted: 4 April 2017

\section{Published online: 24 April 2017}

\section{References}

1. Mills RE, Walter K, Stewart C, Handsaker RE, Chen K, Alkan C, Abyzov A, Yoon SC, Ye K, Cheetham RK, et al. Mapping copy number variation by population-scale genome sequencing. Nature. 2011;470(7332):59-65.

2. Sudmant PH, Rausch T, Gardner EJ, Handsaker RE, Abyzov A, Huddleston J, Zhang Y, Ye K, Jun G, Fritz MHY, et al. An integrated map of structural variation in 2,504 human genomes. Nature. 2015;526(7571):75-+.

3. Batzer MA, Deininger PL. Alu repeats and human genomic diversity. Nat Rev Genet. 2002;3(5):370-9

4. Dewannieux M, Esnault C, Heidmann T. LINE-mediated retrotransposition of marked Alu sequences. Nat Genet. 2003;35(1):41-8.

5. Feng Q, Moran JV, Kazazian Jr HH, Boeke JD. Human L1 retrotransposon encodes a conserved endonuclease required for retrotransposition. Cell. 1996:87(5):905-16.

6. Moran JV, Holmes SE, Naas TP, DeBerardinis RJ, Boeke JD, Kazazian Jr $\mathrm{HH}$. High frequency retrotransposition in cultured mammalian cells. Cell. 1996;87(5):917-27.

7. Konkel MK, Walker JA, Hotard AB, Ranck MC, Fontenot CC, Storer J, Stewart C, Marth GT, Genomes C, Batzer MA. Sequence analysis and characterization of active human Alu subfamilies based on the 1000 genomes pilot project. Genome Biol Evol. 2015;7(9):2608-22.

8. Cost GJ, Boeke JD. Targeting of human retrotransposon integration is directed by the specificity of the $\mathrm{L} 1$ endonuclease for regions of unusual DNA structure. Biochemistry. 1998;37(51):18081-93.

9. Repanas K, Zingler N, Layer LE, Schumann GG, Perrakis A, Weichenrieder O Determinants for DNA target structure selectivity of the human LINE-1 retrotransposon endonuclease. Nucleic Acids Res. 2007;35(14):4914-26.

10. Jurka J. Sequence patterns indicate an enzymatic involvement in integration of mammalian retroposons. Proc Natl Acad Sci U S A. 1997;94(5):1872-7.

11. Xing J, Zhang Y, Han K, Salem AH, Sen SK, Huff CD, Zhou Q, Kirkness EF, Levy S, Batzer MA, et al. Mobile elements create structural variation: analysis of a complete human genome. Genome Res. 2009:19(9):1516-26.

12. Witherspoon DJ, Xing J, Zhang Y, Watkins WS, Batzer MA, Jorde LB. Mobile element scanning (ME-Scan) by targeted high-throughput sequencing. BMC Genomics. 2010;11:410

13. Hormozdiari F, Alkan C, Ventura M, Hajirasouliha I, Malig M, Hach F, Yorukoglu D, Dao P, Bakhshi M, Sahinalp SC, et al. Alu repeat discovery and characterization within human genomes. Genome Res. 2011;21(6):840-9.

14. Stewart C, Kural D, Stromberg MP, Walker JA, Konkel MK, Stutz AM, Urban AE, Grubert F, Lam HY, Lee WP, et al. A comprehensive map of mobile element insertion polymorphisms in humans. PLoS Genet. 2011;7(8):e1002236.

15. Bennett EA, Coleman LE, Tsui C, Pittard WS, Devine SE. Natural genetic variation caused by transposable elements in humans. Genetics. 2004;168(2):933-51.

16. Bennett EA, Keller H, Mills RE, Schmidt S, Moran JV, Weichenrieder O, Devine SE. Active Alu retrotransposons in the human genome. Genome Res. 2008;18(12):1875-83.

17. Payer LM, Steranka JS, Yang WR, Kryatova MS, Medabalimi S, Ardeljan D, Liu C, Boeke JD, Avramopoulos D, Burns KH. Structural variants caused by Alu insertions are associated with risks for many human diseases. Proc Natl Acad Sci USA. 2017. Pending.

18. Johanning K, Stevenson CA, Oyeniran OO, Gozal YM, Roy-Engel AM, Jurka J, Deininger PL. Potential for retroposition by old Alu subfamilies. J Mol Evol. 2003;56(6):658-64.

19. Shen MR, Batzer MA, Deininger PL. Evolution of the master Alu gene(s). J Mol Evol. 1991:33(4):311-20.

20. Smit AFA, Hubley R, Green P. RepeatMasker Open-4.0.6. 2013-2016. http:// www.repeatmasker.org.

21. Bao WD, Kojima KK, Kohany O. Repbase update, a database of repetitive elements in eukaryotic genomes. Mob DNA. 2015;6:6.

22. Lee E, Iskow R, Yang L, Gokcumen O, Haseley P, Luquette 3rd L, Lohr JG, Harris CC, Ding L, Wilson RK, et al. Landscape of somatic retrotransposition in human cancers. Science. 2012;337(6097):967-71.

23. Srikanta D, Sen SK, Huang CT, Conlin EM, Rhodes RM, Batzer MA. An alternative pathway for Alu retrotransposition suggests a role in DNA double-strand break repair. Genomics. 2009;93(3):205-12.

24. Salem AH, Kilroy GE, Watkins WS, Jorde LB, Batzer MA. Recently integrated Alu elements and human genomic diversity. Mol Biol Evol. 2003;20(8):1349-61.
25. Callinan PA, Wang J, Herke SW, Garber RK, Liang P, Batzer MA. Alu retrotransposition-mediated deletion. J Mol Biol. 2005;348(4):791-800.

26. Srikanta D, Sen SK, Conlin EM, Batzer MA. Internal priming: an opportunistic pathway for L1 and Alu retrotransposition in hominins. Gene. 2009;448(2):233-41.

27. Roy-Engel AM, Salem AH, Oyeniran OO, Deininger L, Hedges DJ, Kilroy GE, Batzer MA, Deininger PL. Active alu element "A-tails,": Size does matter. Genome Res. 2002;12(9):1333-44.

28. Xing J, Hedges DJ, Han K, Wang H, Cordaux R, Batzer MA. Alu element mutation spectra: molecular clocks and the effect of DNA methylation. J Mol Biol. 2004;344(3):675-82.

29. Batzer MA, Kilroy GE, Richard PE, Shaikh TH, Desselle TD, Hoppens $C L$, Deininger PL. Structure and variability of recently inserted Alu family members. Nucleic Acids Res. 1990;18(23):6793-8.

30. Carroll ML, Roy-Engel AM, Nguyen SV, Salem AH, Vogel E, Vincent B, Myers J. Ahmad Z, Nguyen L, Sammarco M, et al. Large-scale analysis of the Alu Ya5 and Yb8 subfamilies and their contribution to human genomic diversity. J Mol Biol. 2001;311(1):17-40.

31. Labuda D, Striker G. Sequence conservation in Alu evolution. Nucleic Acids Res. 1989;17(7):2477-91.

32. Xing J, Salem AH, Hedges DJ, Kilroy GE, Watkins WS, Schienman JE, Stewart $C B$, Jurka J, Jorde LB, Batzer MA. Comprehensive analysis of two Alu Yd subfamilies. J Mol Evol. 2003:57 Suppl 1:S76-89.

33. Conti A, Carnevali D, Bollati V, Fustinoni S, Pellegrini M, Dieci G. Identification of RNA polymerase III-transcribed Alu loci by computational screening of RNASeq data. Nucleic Acids Res. 2015;43(2):817-35.

34. Ahl V, Keller H, Schmidt S, Weichenrieder O. Retrotransposition and crystal structure of an Alu RNP in the ribosome-stalling conformation. Mol Cell. 2015;60(5):715-27.

35. Boeke JD. LINEs and Alus-the polyA connection. Nat Genet. 1997;16(1):6-7.

36. van de Lagemaat LN, Gagnier L, Medstrand P, Mager DL. Genomic deletions and precise removal of transposable elements mediated by short identical DNA segments in primates. Genome Res. 2005;15(9):1243-9.

37. Han KD, Xing J, Wang H, Hedges DJ, Garber RK, Cordaux R, Batzer MA. Under the genomic radar: the stealth model of Alu amplification. Genome Res. 2005;15(5):655-64.

38. Teugels E, De Brakeleer S, Goelen G, Lissens W, Sermijn E, De Greve J. De novo Alu element insertions targeted to a sequence common to the BRCA1 and BRCA2 genes. Hum Mutat. 2005;26(3):284.

39. Dewannieux $M$, Heidmann T. Role of poly(A) tail length in Alu retrotransposition. Genomics. 2005;86(3):378-81.

40. Mills RE, Bennett EA, Iskow RC, Devine SE. Which transposable elements are active in the human genome? Trends Genet. 2007;23(4):183-91.

41. Witherspoon DJ, Zhang Y, Xing J, Watkins WS, Ha H, Batzer MA, Jorde LB. Mobile element scanning (ME-Scan) identifies thousands of novel Alu insertions in diverse human populations. Genome Res. 2013;23(7):1170-81.

42. Wang J, Song L, Grover D, Azrak S, Batzer MA, Liang P. dbRIP: a highly integrated database of retrotransposon insertion polymorphisms in humans. Hum Mutat. 2006:27(4):323-9.

43. Iskow RC, McCabe MT, Mills RE, Torene S, Pittard WS, Neuwald AF, Van Meir $E G$, Vertino PM, Devine SE. Natural mutagenesis of human genomes by endogenous retrotransposons. Cell. 2010;141(7):1253-61.

44. Shukla R, Upton KR, Munoz-Lopez M, Gerhardt DJ, Fisher ME, Nguyen T, Brennan PM, Baillie JK, Collino A, Ghisletti S, et al. Endogenous retrotransposition activates oncogenic pathways in hepatocellular carcinoma. Cell. 2013;153(1):101-11.

45. Welter D, MacArthur J, Morales J, Burdett T, Hall P, Junkins H, Klemm A, Flicek P, Manolio T, Hindorff L, et al. The NHGRI GWAS Catalog, a curated resource of SNP-trait associations. Nucleic Acids Res. 2014;42(Database issue):D1001-6.

46. Edgar RC. MUSCLE: multiple sequence alignment with high accuracy and high throughput. Nucleic Acids Res. 2004;32(5):1792-7.

47. Crooks GE, Hon G, Chandonia JM, Brenner SE. WebLogo: a sequence logo generator. Genome Res. 2004;14(6):1188-90 